\title{
A case of leiomyoma of the oesophagus complicated by superior vena cava obstruction and associated eosinophilia
}

\author{
P. HICKLING \\ B.Sc., M.R.C.P.
}

K. BuKSH

M.B. B.S.

\author{
P. BECK \\ M.D., M.R.C.P.
}

Departments of Medicine and Thoracic Surgery, Llandough Hospital, and University Hospital of Wales, Cardiff

\section{Summary}

Superior vena cava obstruction occurring as a complication of leiomyoma of the oesophagus has not been reported before. Such a case is recorded which was associated with striking eosinophilia, a feature previously noted in cases of uterine leiomyomas.

\section{Case report}

A 35-year-old merchant seaman had complained of intermittent epigastric pain, water brash and heartburn for approximately a year. These symptoms were usually associated with drinking bouts in which he frequently and freely indulged, but which he had recently curtailed. He had 3 episodes of haematemesis during the year, each being associated with drinking. For 6 months he had noticed increasing dysphagia for solids and he occasionally regurgitated solid food. In March 1978 he had a barium meal examination which showed a smooth compression of the oesophagus measuring $7 \mathrm{~cm} \times 5 \mathrm{~cm}$ posteriorly and to the right side of the aortic arch (Fig. 1). This investigation was repeated with tomography and confirmed a large extrinsic impression on the right side of the oesophagus. No hilar gland enlargement was identified. At this time his white cell count was $8 \cdot 2 \times 10^{9} / 1$, with a normal differential. He was awaiting further investigation when in May he presented as an emergency with a 6-day history of increasing swelling of his left arm, left hand and his face.

On examination, there was pitting oedema of the dorsum of his left hand and a firm swelling of the whole of his left arm. Both supra-clavicular fossae and the suprasternal notch were obliterated. There was swelling over the neck, face and anterior chest wall, and moderate conjunctival suffusion. The jugular veins were distended to the level of the ear lobes and were non-pulsatile. The retinal veins were

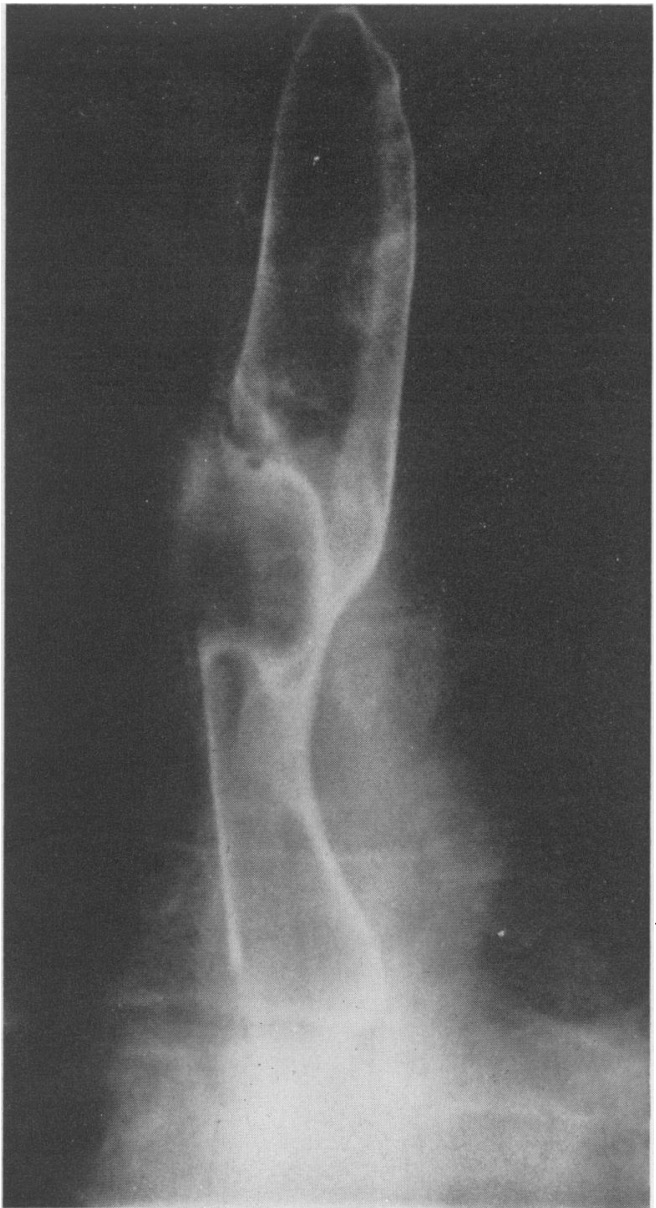

Fig. 1. Barium meal examination-the leiomyoma is seen compressing the right side of the oesophagus. 
engorged but there was no papilloedema. General examination was normal and in particular there was no lymphadenopathy. His peripheral pulses were normal, blood pressure $120 / 70 \mathrm{mmHg}$ in both arms, and there were no abnormal signs in the chest or abdomen.

Investigations at that time were as follows: $\mathrm{Hb}$, $16.6 \mathrm{~g} / \mathrm{dl}$; white cell count, $23.1 \times 10^{9} / 1 ;(15 \%$ eosinophils, $66 \%$ neutrophils, $15 \%$ lymphocytes, $1 \%$ monocytes and $3 \%$ myelocytes).

Bone marrow cytology showed a highly cellular marrow with a striking degree of eosinophilia, constituting $65 \%$ of the total cells of which eosinophil myelocytes constituted $20 \%$, eosinophil metamyelocytes $40 \%$ and mature eosinophils $40 \%$ of the total eosinophil count. His Mantoux test was negative at a dilution of $1 / 10000$.

\section{Management and progress}

It was decided to treat his superior vena caval obstruction with steroids. He was given prednisone $40 \mathrm{mg} / \mathrm{day}$, and the signs in his face, neck and left arm resolved completely over the next $48 \mathrm{hr}$. The eosinophilia increased over this period with the WCC rising to $36.5 \times 10^{9} / 1$, of which $28 \%$ were eosinophils. Upper gastro-intestinal endoscopy was performed after the superior vena caval obstruction had settled. This revealed a smooth swelling approximately $3 \mathrm{~cm}$ in diameter, impinging on the oesophageal lumen at $25 \mathrm{~cm}$. The mucosa overlying it was normal and there was no pulsation visible. At $35 \mathrm{~cm}$ there was a sliding hiatus hernia. The endo- scope passed easily into the stomach which waš normal in appearance.

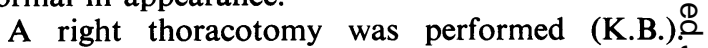
There was no mediastinal lymphadenopathy butthere was a round, shiny tumour, $6.5 \mathrm{~cm}$ in diameter $\overrightarrow{\overrightarrow{2}}$ within the right antero-lateral wall of the oesophagus The trachea was deviated to the right and the tumour was bulging forwards closely adjacent to the junction of the innominate veins, the azygos vein and the superior vena cava (Fig. 2). The tumour was easily? enucleated from the oesophageal muscle leaving thes mucosa intact. Treatment with prednisone wass stopped after 2 weeks.

\section{Histology of tumour}

Macroscopic appearance - a rounded encapsulated tumour, rubbery in consistency. The cut surface was glistening and white with areas of degeneration ap both poles.

Microscopic appearance - the appearance was tha of a leiomyoma with areas of necrosis. There were many focal and perivascular collections of eosinoo phils (Fig. 3).

The patient made an uneventful recovery. Post $\vec{\varepsilon}$ operative barium swallow showed normal oesopha geal motility with no evidence of leakage. The patient was soon swallowing normally. His differen blood count returned to normal. A superior vera cavagram with injection of contrast into both arôn was performed 2 weeks after surgery and this demo strated patent central veins with no evidence of invasion, displacement or thrombus. He remains๊

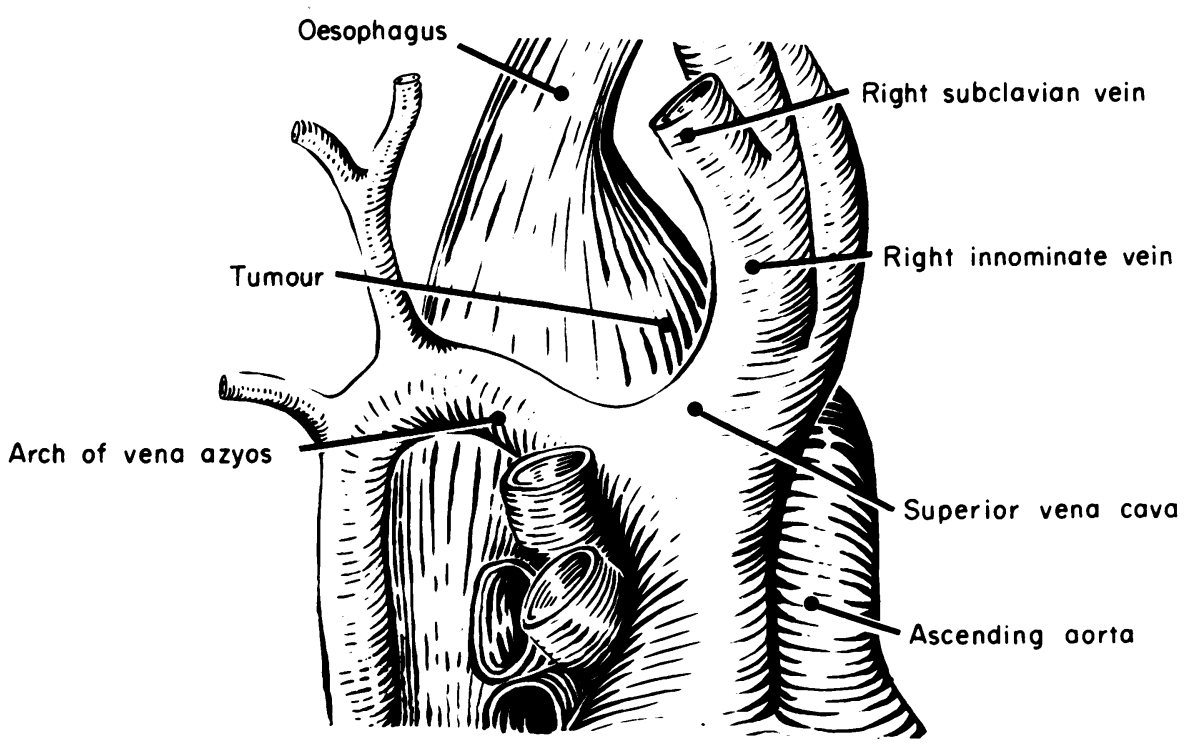

FIG. 2. Diagram showing position of leiomyoma as seen at right thoracotomy. 


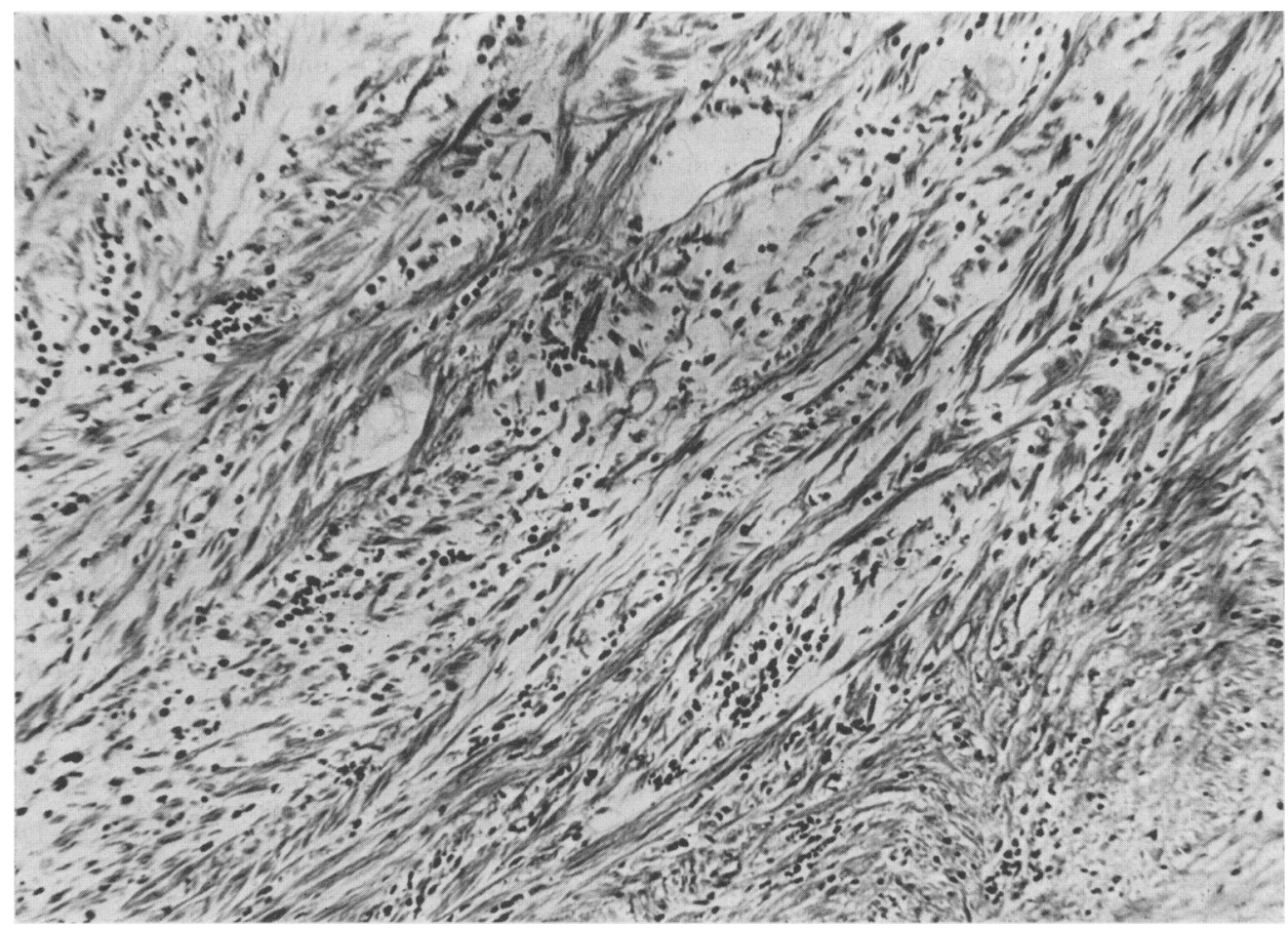

FIG. 3. Photomicrograph of leiomyoma showing intense infiltrate of eosinophils $(\times 80)$.

perfectly fit, with no recurrence of his superior vena caval obstruction and his peripheral blood count is entirely normal, 6 months after removal of the oesophageal leiomyoma.

\section{Discussion}

Leiomyomas are the commonest benign tumours of the oesophagus (Seremetis et al., 1975; Mansour, Hatcher and Huan, 1977). Symptoms, if any, are usually confined to the local effects of pressure on the oesophageal lumen, such as dysphagia and chest pain. Acid reflux, usually seen in cases with associated hiatus hernia, as in this case, is also a common complaint. Weight loss, presumably due to dysphagia, is a less common symptom. Complications of oesophageal leiomyomas are rare, but malignant change, haemorrhage and asphyxia have all been reported (Seremetis et al., 1975). So far as the authors know, this is the first case in which potentially lethal obstruction of the superior vena cava has been reported. No other lesion could be found to account for the obstruction either radiologically, endoscopically or at surgery.

It was initially suspected that the patient had a mediastinal malignancy, possibly a lymphoma, in view of the peripheral blood eosinophilia. The prompt response of the obstruction to steroids strengthened these suspicions, but whether this response was coincidental or due to a direct local effect on the tumour and/or associated oedema of the surrounding tissues, is not known. No other cause for the eosinophilia was discovered despite an exhaustive search. He was not taking any drugs known to cause eosinophilia.

The most common haematological abnormality associated with leiomyomas, iron-deficiency anaemia excepted, is polycythaemia (Payne, Woods and Wrigley, 1969). A leucocytosis in the presence of an infected and/or necrotic leiomyoma sometimes occurs (Buka, 1965). Although well recognized in association with malignancy, eosinophilia is only rarely associated with benign tumours (Isaacson and Rapaport, 1946; Murray, 1953). Buka (1965) reported 2 cases of uterine leiomyomas associated with eosinophilia which resolved after hysterectomy. In neither case was the eosinophilia as marked as in the present case. The extensive eosinophilic infiltration of the tumour itself is also interesting in this case with respect to the peripheral blood and bone marrow eosinophilia. In the cases reported by Buka, 
no areas of acute or chronic inflammation were present in the uteri, nor was there any collection of eosinophils in the leiomyomas themselves, and no report is made of areas of necrosis, in contradistinction to the histological findings in the present case. The stimulus for the eosinophilia in all 3 cases must remain conjectural but, like the polycythaemia sometimes associated with leiomyomas, the eosinophilia has promptly resolved when the leiomyomas were removed (Rothman and Rennard, 1963). Eosinophilic infiltration of leiomyomas is not a feature which has hitherto been noted but eosinophilic infiltration of necrotic malignant tumours has been reported (Isaacson and Rapaport, 1946). It is thought unlikely, however, that in the case of leiomyomas, the necrosis alone is responsible for the eosinophilia, as necrotic degeneration, often associated with haemorrhage, is a common event in leiomyomas of the uterus and eosinophilia is not usually seen in these cases. Buka speculates that in his cases the eosinophilia is due to an auto-immune response to the leiomyomas which is manifested by the eosinophilia. It is not clear how this autoimmune response may be mediated but it is an attractive hypothesis to explain the puzzling association between leiomyomas and eosinophilia. The present authors can only conclude, as Buka does, that leiomyomas should be included in the differen tial diagnosis of any unexplained eosinophilia.

It appears, therefore, that the present case is one of leiomyoma of the oesophagus complicated by 4 superior vena cava obstruction and associated with massive eosinophilia involving the tumour itself the bone marrow and the peripheral blood.

\section{References}

BUKA, N.J. (1965) Eosinophilia associated with uterinen leiomyomas. Canadian Medical Association Journal, 93, 163.

ISAACSON, N.H. \& RAPAPORT, P. (1946) Eosinophilia in malignant tumors: its significance. Annals of Internat $\vec{\omega}$ Medicine, 25, 893.

Mansour, K.A., Hatcher, C.R. \& Huan, C.L. (1977)르 Benign tumors of the esophagus: Experience with 20 cases. Southern Medical Journal, 70, 461.

MurRaY, R.C. (1953) The use of the absolute eosinophifr count in the diagnosis of neoplasms. New England Journab of Medicine, 248, 848.

Payne, P., Woods, H.F. \& Wrigley, P.F.M. (1969) Uterine fibromyomata and secondary polycythaemia. Journal of Obstetrics and Gynaecology of the British Commonwealth, 76, 845.

Rothman, D. \& RenNaRd, M. (1963) Myoma-erythrocytosis_syndrome. Obstetrics and Gynecology, 21, 210.

Seremetis, M.G., Lyons, W.S., DeGuzman, V.C. \& Peabody, J.W. (1976) Leiomyomata of the esophagus. An analysisD of 838 cases. Cancer. New York, Philadelphia, etc., 38, 2166. 